\title{
Non-Linear EH Relaying in Delay-Transmission Mode over $\eta-\mu$ Fading Channels
}

\author{
Ayaz Hussain ${ }^{\dagger 1}$, Inayat $\mathrm{Ali}^{\dagger 2}$, Ramesh Kumar ${ }^{\dagger \dagger}$, Zahoor Ahmed ${ }^{\dagger 4}$ \\ ${ }^{\dagger}$ Department of Electrical Engineering, Balochistan University of Engineering \& Technology, Khuzdar, Pakistan \\ ${ }^{\dagger \dagger}$ Department of Electronic Engineering, Dawood University of Engineering \& Technology, Karachi, Pakistan
}

\begin{abstract}
Energy harvesting is a technique to harvest energy from RF (radio frequency) waves. The RF signals have the ability to convey energy and information concurrently. The EH in cooperative relaying systems may increase the capacity and coverage of wireless networks. In this work, we study a dual-hop (two-hop) relaying. This system has three nodes: a source, a relay, and a destination. The source and destination have multiple antennas. We account a non-linear EH model and TSR (time-switchingbased relaying) protocol at the single-antenna relay node. We evaluate the system performance over $\eta-\mu$ fading channels. With a saturation threshold, a non-linear EH receiver restrains the harvested power. In the TSR protocol, the relay changes mode between the EH and information processing, by which a fraction of time is used with each process. The fading model $\eta-\mu$ incorporates some fading models as notable cases, viz., Nakagami$m$, One-sided Gaussian, Nakagami- $q$ (Hoyt), and Rayleigh. The system performance is analyzed in terms of the average capacity and throughput for different saturation threshold power levels, divers antennas arrangements, and different parameter values of $\eta$ and $\mu$.
\end{abstract}

Keywords-EH relay; non-linear EH model; $\eta-\mu$ fading; TSR protocol; throughput

\section{INTRODUCTION}

Energy harvesting $(\mathrm{EH})$ is a technology to gather energy from the surrounding radio frequency (RF) waves and got outstanding recognition to sustain a network lifetime [1][14]. The RF signals have an ability to convey energy and information concurrently, therefore, it is possible to collect or harvest the energy from the RF waves and that harvested energy can easily be reserved or used for electronic equipment to work [6].

RF-based EH technology is studied in cooperative relaying networks [2]-[14] (and references therein), where from the received RF signals, a relay node collects energy. Dual-hop (or Two-hop) relaying is a popular technique to obtain greater capacity and larger coverage of wireless networks [14]. There are two well-known methods for relaying data: AF (amplifyand-forward) method and DF (decode-and forward) method. In an AF relay method, a relay of a dual-hop system amplifies the received message of the source node and forwards it to the next receiver or destination. In a DF relay method, the received message at the relay node is decoded first then forwarded to the next receiver or destination. Recently, a lot of research papers are written on energy harvesting in a two-hop DF relaying system in the literature [2]-[14].

There are two main EH protocols for two-hop relaying systems: PSR (power-splitting-based relaying) protocol and
TSR (time switching-based relaying) protocol. The relay alternatively splits the received signal power from the source node and time into two parts in the PSR and TSR protocols, respectively [6]. A non-linearity of an $\mathrm{EH}$ relay/receiver restrict the level of the harvested energy because it is not a practical node. The performance of a two-hop DF EH relaying network based on a non-linear mode of $\mathrm{EH}$ receiver was investigated for classical fading channels in [6]-[12] and for general $\kappa-\mu$ shadowed and $\eta-\mu$ fading channels, respectively, in [6] and [13]. In [6], a DF EH relaying system was studied based on non-linear $\mathrm{EH}$ receiver with hardware impairments and performance was analyzed under $\kappa-\mu$ shadowed fading channels. In [7], the performance of an AF EH relaying system with a non-linear energy harvester for Nakagami- $m$ fading channels was analyzed. A partial DF relay selection scheme with a non-linear energy harvester was investigated in [8]. In [9], using a non-linear EH receiver model, a two-hop relaying system was investigated where multiple-antennas were installed at the destination and source only and performance was analyzed for a different number of antennas. In interferencelimited environments of Nakagami- $m$, the DF relaying system performance with a non-linear energy harvester is analyzed in [10]. In [11], the authors investigated the secrecy performance for a two-hop DF relaying with a non-linear energy harvester. In this system, the best relay is selected using CSI (channel state information) which assists the source to send its message signal to the destination. In [12], an AF non-linear EH relaying system was studied with perfect and imperfect CSI. Recently, a non-linear EH relay receiver in conjunction with the energy harvesting PSR protocol in a two-hop EH relaying for $\eta-\mu$ fading channels was examined in a delay-limited transmission mode [13].

Despite the importance of a delay-tolerant transmission mode and a non-linear $\mathrm{EH}$ relay receiver in a two-hop $\mathrm{EH}$ cooperative relaying network, the impact on the system performance owing to a non-linear model of $\mathrm{EH}$ receiver node (i.e., EH relay receiver) with a TSR protocol in a delay-tolerant transmission mode under $\eta-\mu$ fading channels is not studied yet.

In this paper, in a delay-tolerant transmission mode, the impact on the system performance due to a non-linearity of $\mathrm{EH}$ receiver in a two-hop $\mathrm{EH}$ relaying is investigated under $\eta-\mu$ fading environments. We consider a TSR method [4] to analyze the system performance in $\eta-\mu$ fading environments. The fading model $\eta-\mu$ is a general model, therefore, from this fading model, some special cases can be obtained with special parameters, viz. Rayleigh, One-sided Gaussian, Hoyt, and Nakagami- $m$ [15]. Therefore, from our results which are 
obtained using the general $\eta-\mu$ fading channels, we can figure out some identical and non-identical cases, such as, the Rayleigh/Rayleigh, One-sided Gaussian/One-sided Gaussian, Nakagami- $m /$ Nakagami- $m$, Hoyt/Hoyt, and combinations of such fading links.

The remaining sections of our paper are sectioned as follows: In Section II, firstly, we give a brief introduction of the considered system model and then we describe the $\eta-\mu$ channel model and its particular cases; in Section III, performance analysis of the assumed system is provided; the identical and non-identical fading cases which are obtained from the $\eta-\mu$ fading scenario are discussed in Section IV; in Section V, based on the obtained expressions, the numerical as well simulated results are given; in the last section, the conclusion of our paper is concluded.

\section{System AND Channel Models}

\section{A. System Model}

We account a two-hop relaying system which is exhibited in Fig. 1. This system has three nodes, namely a source node $S$ which transmit signals to a relay, a relay node $\mathrm{R}$ that transmits received signal to a destination, and a destination node $\mathrm{D}$ which receives signals of the source node via the relay node. The multiple-antennas, $N_{1}$ and $N_{2}$, respectively, are installed at $\mathrm{S}$ and D. A single-antenna relay node has no external source of energy, hence, it harvests or generates energy from the obtained $\mathrm{RF}$ waves from the source node and utilizes that generated power to send the information of $\mathrm{S}$ to $\mathrm{D}$. A TSR protocol is considered at the relay node [4]. Let $\mathbf{h}_{1}$ and $\mathbf{h}_{2}$ are the $N_{1} \times 1$ and $1 \times N_{1}$ channel vectors, respectively, of $\mathrm{S}$ to $\mathrm{R}$ and $\mathrm{R}$ to $\mathrm{D}$. For the TSR protocol, the transmission block structure

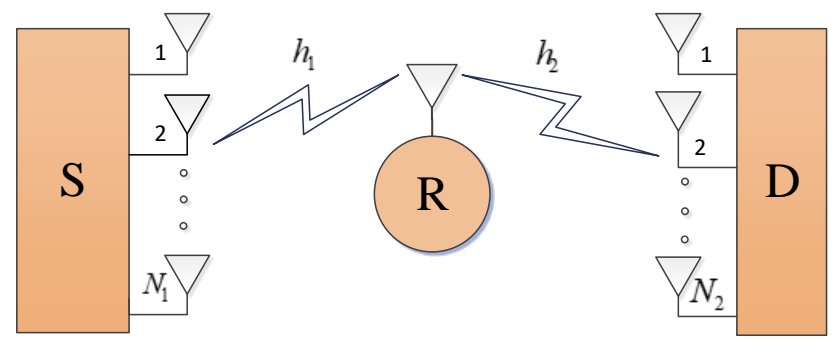

Fig. 1. System model

for information processing and $\mathrm{EH}$ is shown in Fig. 2 [4], where block time is designated by $T$. In the block time $T, \mathrm{~S}$ transfers the message to $\mathrm{D}$ and $\alpha$ shows the fragment of the block time. In a TSR method, R node alternatively switches the received signal in $\alpha:(1-\alpha) T$ proportion. The node $\mathrm{R}$ harvests energy in the fragment of $\alpha$ and the rest fragment $(1-\alpha) T$ is separated into two sub-portions; $(1-\alpha) T / 2$ is employed for $\mathrm{S}$ to $\mathrm{R}$ communication and $(1-\alpha) T / 2$ is employed for $\mathrm{R}$ to $\mathrm{D}$ communication. In line with the TSR protocol, the received signals are sent to the information processor and energy harvester for time $(1-\alpha) T / 2$ and $\alpha T$, respectively. The harvested energy for $\alpha T$ is obtained as [8]

$$
E_{h}=\zeta P_{s}\left\|\mathbf{h}_{1}\right\|^{2} \alpha T
$$

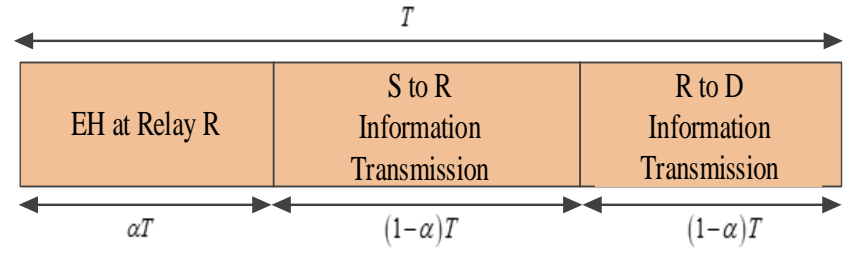

Fig. 2. In the TSR scheme, transmission block structure for EH and information processing [4].

where $\zeta$ designates the efficiency of energy conversion and $P_{s}$ is the power which is transmitted by the source.

We consider a non-linear $\mathrm{EH}$ receiver. When the input power of a non-linear EH receiver is above the saturation level of the threshold power $P_{t h}$, it produces a sustained transmit power $\zeta P_{t h}[6]$. Thus, the relay transmit power, $P_{r}$, can be written as [6]

$$
P_{r}=\frac{2 \zeta \alpha}{(1-\alpha)} \min \left(P_{s}\left\|\mathbf{h}_{1}\right\|^{2}, P_{t h}\right) .
$$

The SNR (signal-to-noise ratio) at the relay node and destination node, are respectively, given by [4] [8]

$$
\gamma_{R}=\frac{P_{s}\left\|\mathbf{h}_{1}\right\|^{2}}{\sigma_{a, r}^{2}+\sigma_{c, r}^{2}}
$$

and

$$
\gamma_{D}= \begin{cases}\frac{2 \alpha \zeta P_{s}\left\|\mathbf{h}_{1}\right\|^{2}\left\|\mathbf{h}_{2}\right\|^{2}}{(1-\alpha)\left(\sigma_{a, d}^{2}+\sigma_{c, d}^{2}\right)}, & P_{s}\left\|\mathbf{h}_{1}\right\|^{2} \leq P_{t h}, \\ \frac{2 \alpha \zeta P_{t h}\left\|\mathbf{h}_{1}\right\|^{2}}{(1-\alpha)\left(\sigma_{a, d}^{2}+\sigma_{c, d}^{2}\right)}, & P_{s}\left\|\mathbf{h}_{1}\right\|^{2}>P_{t h},\end{cases}
$$

where $\sigma_{a, r}^{2}$ and $\sigma_{c, r}^{2}$ are the noise and convergence variances at the relay node, respectively. Additionally, $\sigma_{a, d}^{2}$ and $\sigma_{c, d}^{2}$ are the noise and convergence variances at the destination node, respectively.

TABLE I. ThE $\eta-\mu$ DistRibution AND ITS SPECIAL CASES [13]

\begin{tabular}{lcc}
\hline Distribution & $\eta$ & $\mu$ \\
\hline Rayleigh & $\eta \rightarrow 0$ & $\mu=0.5$ \\
Nakagami- $m$ & $\eta \rightarrow 1$ & $\mu=m / 2$ \\
Hoyt & $\eta \rightarrow q^{2}$ & $\mu=0.25$ \\
\hline
\end{tabular}

\section{B. The $\eta-\mu$ Channel Model}

The $\eta-\mu$ is a general fading model. This fading model incorporates few fading models as notable cases, viz., Nakagami$m$, Onw sided Gaussian, Nakagami- $q$ (Hoyt), and Rayleigh [15]. Let $\gamma_{\ell}(\ell=1,2)$ is the instantaneous SNR of the $\ell$ th link. The PDF (probability density function) of $\gamma_{\ell}(\ell=1,2)$ cab be written as $[15$, eq. (3)]

$$
\begin{aligned}
f_{\gamma_{\ell}}(\gamma)= & \frac{2 \sqrt{\pi} h_{\ell}^{N_{\ell} \mu_{\ell}}}{\Gamma\left(N_{\ell} \mu_{\ell}\right) H_{\ell}^{N_{\ell} \mu_{\ell}-0.5}}\left(\frac{\mu_{\ell}}{\bar{\gamma}_{\ell}}\right)^{N_{\ell} \mu_{\ell}+0.5} \gamma^{N_{\ell} \mu_{\ell}-0.5} \\
& \times \exp \left(\frac{2 \mu_{\ell} h_{\ell}}{\bar{\gamma}_{\ell}} \gamma\right) I_{N_{\ell} \mu_{\ell}-0.5}\left(2 \frac{\mu_{\ell} H_{\ell}}{\bar{\gamma}_{\ell}} \gamma\right),
\end{aligned}
$$


herein, $\eta_{\ell}$ and $\mu_{\ell}$ are the fading parameters, $I_{\mathrm{v}}(\cdot)$ denotes the $\mathrm{v}$-th order of the modified Bessel function of the 1st kind, $h_{\ell}=\left(2+\eta_{\ell}^{-1}+\eta_{\ell}\right) / 4, H_{\ell}=\left(\eta_{\ell}^{-1}-\eta_{\ell}\right) / 4[15], I_{\mathrm{v}}(\cdot)$ denotes the average SNR of the $\ell$-the link, and $\Gamma(\cdot)$ shows the Gamma function.

In Table I, we summarized the special or particular cases of the $\eta-\mu$ fading model where $q$ and $m$ shows the fading parameters of the distributions, respectively, Hoyt and Nakagami- $m$.

\section{Performance ANAlysis}

In this section, firstly, the average capacity is described, then based on the average capacity expression, the achievable throughput is eventually obtained.

\section{A. Average Capacity Analysis}

The statistical mean of the mutual information between the transmitter (i.e., source) and receiver (i.e., destination) is the average capacity. For a DF EH relaying system, the average capacity can be obtained as [6]

$$
\bar{C}=\min \left(\bar{C}_{R}, \bar{C}_{D}\right)
$$

where $\bar{C}_{R}=\frac{1}{2} E\left[\log _{2}\left(1+\gamma_{R}\right)\right], \bar{C}_{R}=\frac{1}{2} E\left[\log _{2}\left(1+\gamma_{D}\right)\right]$, $E[\cdot]$ denotes the expectation operator, and $\gamma_{R}$ and $\gamma_{D}$ are given by (3) and (4), respectively.

\section{B. Throughput Analysis}

The throughput of a dual EH relaying system with beamforming based on non-linear EH receiver in a mode of a delaytolerant transmission is provided as [6]

$$
\tau=\frac{(1-\alpha)}{2} \bar{C} .
$$

Utilizing (7) and with the aid of Matlab, we can acquire the optimal time-switching ratio $\alpha^{*}$ and the optimal throughput $\tau^{*}$ numerically.

\section{Special Cases}

Some particular cases are included in the $\eta-\mu$ fading model, namely Nakagami- $m$, Rayleigh, and Hoyt. Hence, the average capacity and throughput expressions for the different fading cases can be obtained from (6) and (7) with special parameters as given in Table I. Subsequently, the possible fading conditions are Rayleigh/Rayleigh, Rayleigh/Nakagami$m$, Rayleigh/Hoyt, Nakagami- $m /$ Nakagami- $m$, Nakagami$m /$ Rayleigh, Hoyt/Hoyt, Nakagami-m/Hoyt, Hoyt/Rayleigh, and Hoyt/Nakagami- $m$. These special cases are also discussed in [13, Table II] for delay-limited transmission mode.

\section{Numerical Results}

TABLE II. THE VALUES OF PARAMETERS USED IN SIMULATION

\begin{tabular}{cccccc}
\hline & Parameter & Value & & Parameter & Value \\
\hline 1 & $\zeta$ & 0.9 & 5 & $\sigma_{a, r}^{2}$ & $0.045 \mathrm{~W}$ \\
2 & $P_{s}$ & $5 \mathrm{~W}$ & 6 & $\sigma_{c, r}^{2}$ & $0.045 \mathrm{~W}$ \\
3 & $\lambda_{1}$ & $1 \mathrm{~W}$ & 7 & $\sigma_{a, d}^{2}$ & $0.045 \mathrm{~W}$ \\
4 & $\lambda_{2}$ & $1 \mathrm{~W}$ & 8 & $\sigma_{c, d}^{2}$ & $0.045 \mathrm{~W}$ \\
\hline
\end{tabular}

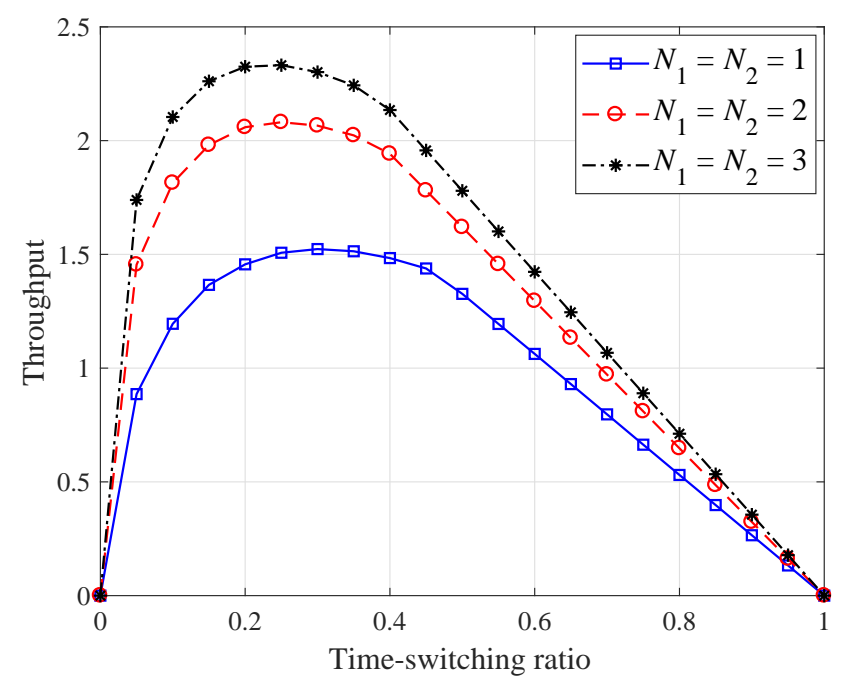

Fig. 3. Throughput against time-switching ratio for different arrangement of antennas when $P_{t h}=3, \mu_{1}=\mu_{2}=1$, and $\eta_{1}=\eta_{2}=0.9$.

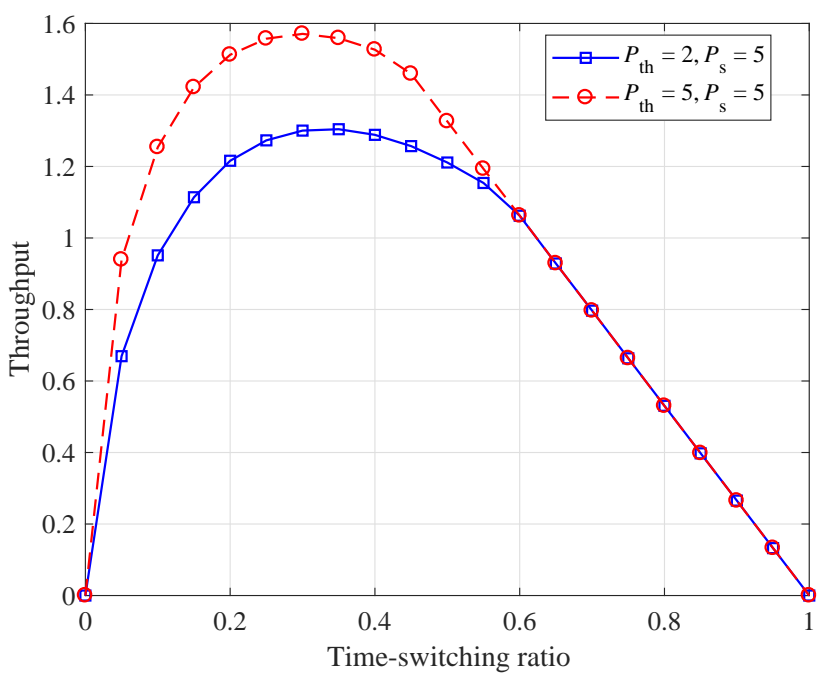

Fig. 4. Throughput against time-switching ratio for saturation threshold power levels, $P_{t h}$, when $\mu_{1}=\mu_{2}=1, \eta_{1}=\eta_{2}=0.9$, and $N_{1}=N_{2}=2$.

Here, in Section V, the performance is evaluated of the twohop EH relaying system that has a non-linear model of EH relay receiver in $\eta-\mu$ fading environment. We set some basic parameters throughout simulations as presented in Table II, unless otherwise stated.

Fig. 3 shows the throughput, $\tau$, against time-switching ratio, $\alpha$, for divers antennas organizations. As expected, the throughput is increased with increasing the number of antennas. The throughput increases as time-switching ratio, $\alpha$, grows from 0 to $\alpha^{*}$ (i.e., a point of optimal-value of the time-switching ratio where the system achieves the maximum throughput), and the value of the throughput lowers as $\alpha$ grows from the optimal-value $\alpha^{*}$ to 1 .

Fig. 4 reveals the average capacity in $\eta-\mu$ fading environment by considering the linear and non-linear energy harvesting receiver when we set $N_{1}=N_{2}=2, \eta_{1}=\eta_{2}=1$, 


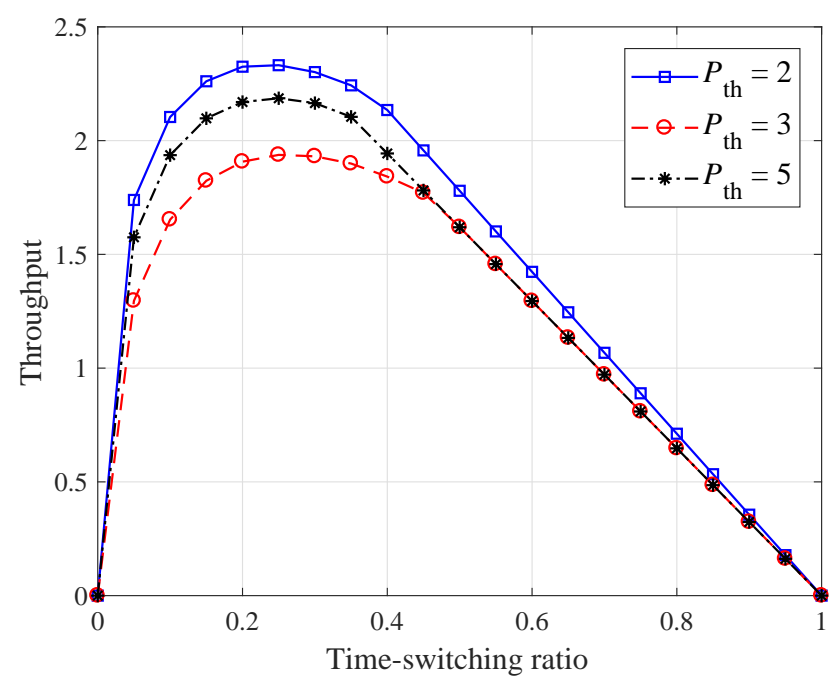

Fig. 5. Throughput against time-switching ratio for saturation threshold power levels, $P_{t h}$, when $\mu_{1}=\mu_{2}=1, \eta_{1}=\eta_{2}=0.9$, and $N_{1}=N_{2}=2$.

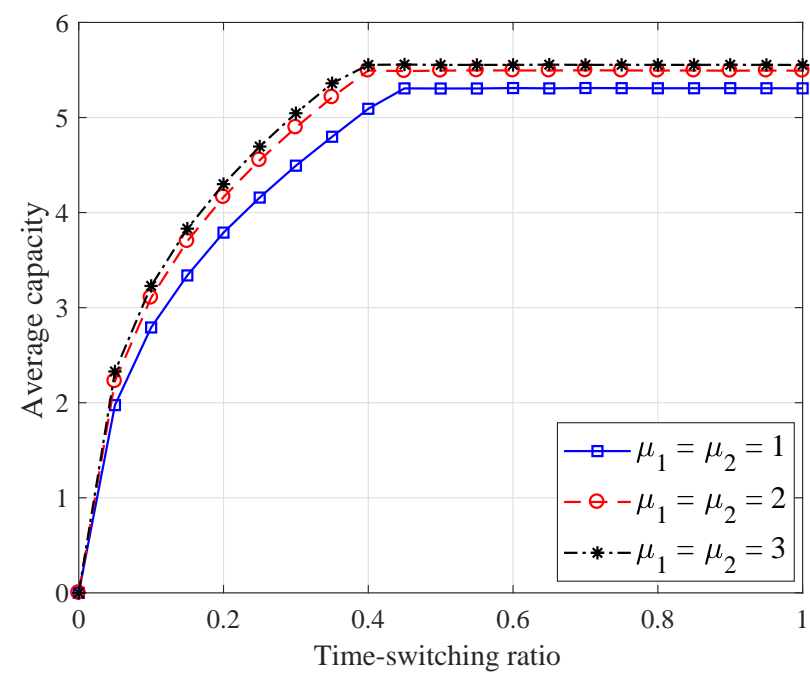

Fig. 6. Average capacity against time-switching ratio for different $\mu$ (i.e., $\mu_{1}$ or $\left.\mu_{2}\right)$ when $P_{t h}=3, N_{1}=2, N_{2}=2, \eta_{1}=1$ and $\eta_{2}=1$.

and $\mu_{1}=\mu_{2}=2$. From Fig. 4. one can notice that the system performance is better with a linear $\mathrm{EH}$ relay receiver as compared to a non-linear EH relay receiver. The receiver for energy harvesting is a non-linear node and yields a sustained transmit power $\zeta P_{t h}$ if the given power to the receiver for energy harvesting is at a higher level than a saturation power $P_{t h}$.

In Fig. 5, the performance based on throughput with respect to time-switching ratio is shown for different saturation threshold power levels. From Fig. 5, it is seen that the performance in terms of throughput improves with the saturation level of threshold power $P_{t h}$. The enhancement in the mount of saturation threshold power decreases the probability of saturation of the $\mathrm{EH}$ receiver; in fact, the $\mathrm{EH}$ receiver of relay need more power to harvest energy.

Fig. 6 exhibits the average capacity performance versus

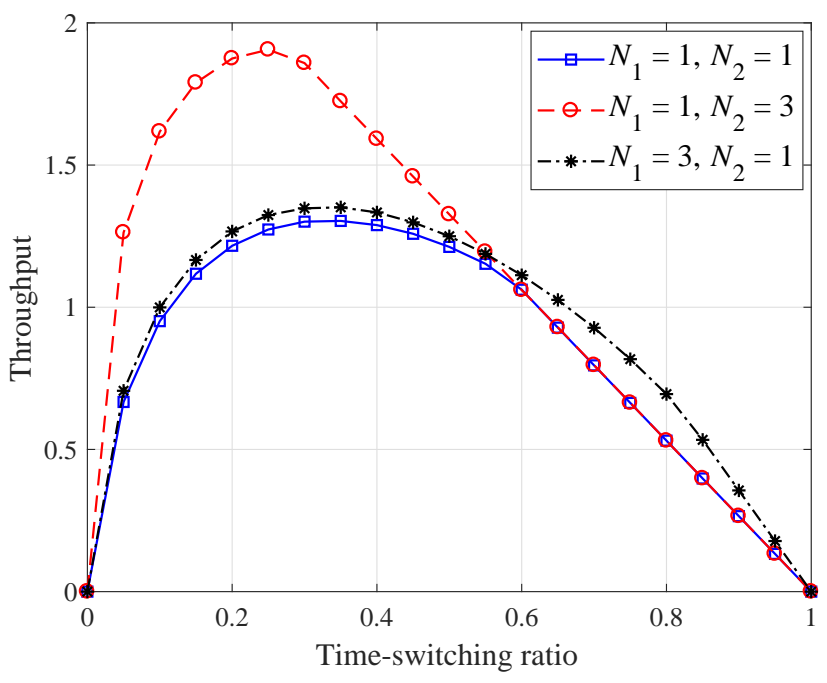

Fig. 7. Throughput performance for various antenna arrangements when $\mu_{1}=\mu_{2}=1, \eta_{1}=\eta_{2}=0.5$, and $P_{t h}=3$.

time-switching ratio for distinct values of $\mu\left(\mu_{1}, \mu_{2}\right)$. From this, we perceive that the increment in parameter $\mu$, subsequently, raises the overall performance.

Fig. 7 shows the throughput performance when we set different number of antennas. In a non-linear EH mode, it is seen that the throughput performance is better when we set $N_{1}<N_{2}$ as compared to $N_{1}<N_{2}$.

\section{CONCLUSION}

A DF EH cooperative relaying with beamforming that has a non-linear $\mathrm{EH}$ relay receiver is studied in $\eta-\mu$ fading environments. We assumed a non-linear $\mathrm{EH}$ relay receiver and time switching TSR protocol at the relay node. With respect to the number of antennas, parameter $\mu$, amount of saturation threshold power, we evaluated our system in different environments of $\eta-\mu$ fading. From our obtained results, we concluded that the effect of the saturation level of threshold power can be reduced with beamforming techniques. The special cases of fading channels can be deduced from the general model of $\eta-\mu$ fading, therefore, we can obtain new analytical results for various classical (Rayleigh, Hoyt, and Nakagami- $m$ ) and general fading conditions.

\section{ACKNOWLEDGMENT}

Our research work is supported and funded by ORIC, Balochistan UET, Khuzdar, Balochistan, Pakistan.

\section{REFERENCES}

[1] X. Lu, P. Wang, D. Niyato, and et al., "Wireless networks with RF energy harvesting: A contemporary survey", IEEE Commun. Surv. Tuttorials., vol. 17, no. 2, pp. 757-789, 2015.

[2] A. Hussain, Z. Ahemd, I. Ali, and S.H., Kim, "Energy harvesting relaying network in a delay-tolerant transmission mode over $\kappa-\mu$ shadowed fading channels", International Journal of Computer Science and Network Security, vol. 18, no. 3, pp. 119-125, 2018.

[3] A. Hussain, Z. Ahmed, U. Rajpoot, and et al., "Energy harvesting relaying network with hardware impairments in $\eta-\mu$ fading environment", International Journal of Computer Science and Network Security, vol. 18, no. 4, 2018. 
[4] A.A, Nasir, X. Zhou, S. Durrani, and R.A, Kennedy, "Throughput and ergodic capacity of wireless energy harvesting based DF relaying network", proc. IEEE ICC 2014, Sydney, Australia, June 10-14, 2014.

[5] O.S. Badarneh, F.S. Almehmadi, I.S. Ansari, and X. Yang, "Wireless energy harvesting in cooperative decode-and-forward relaying networks over mixed generalized $\eta-\mu$ and $\kappa-\mu$ fading channels", Transation on Emerging Telecommunication Technology, DOI: 10.1002/ett.3262, pp.1$18,2017$.

[6] A. Hussain, S.-H. Kim, and S.-H. Chang, "Non-linear energy harvesting relaying with beamforming and hardware impairments in $\kappa-\mu$ shadowed fading environment", Transations on Emerging Telelecommunicaiton Technology, https://doi.org/10.1002/ett.3303, 2018.

[7] Y. Dong, M.J. Hossain, and J. Cheng, "Performance of wireless powered amplify and forward relaying over Nakagami-m fading channels with nonlinear energy harvester", IEEE Commun Lett., vol.20, no.4, pp.672675, 2016.

[8] T.M. Hoang, T.T. Duy, and V.N.Q. Bao, "On the performance of nonlinear wirelessly powered partial relay selection networks over Rayleigh fading channels", proc. 3rd NICS 2016, Danag, Vietnam, September 14-16, 2016,

[9] J. Zhang, and G. Pan "Outage analysis of wireless-powered relaying MIMO systems with non-linear energy harvesters and imperfect CSI",
IEEE ACCESS, vol.4, no., pp.7046-7053, 2016.

[10] A. Cvetkovic, V. Blagojevic, and P. Ivanišs, "Performance analysis of nonlinear energy-harvesting $D F$ relay system in interference-limited Nakagami-m fading environment", ETRI Journal, vol.39, no., pp.803812, 2017.

[11] J. Zhang, G. Pan and Y. Xie "Secrecy outage performance for wirelesspowered relaying systems with nonlinear energy harvesters", Front. Inform. Technol. Electron. Engg., vol.18, no.2, pp.246-252, 2017.

[12] K. Wang, Y. Li, Y. Ye, and H. Zhang "Dynamic power splitting schemes for non-Linear EH relaying networks: perfect and imperfect CSI", Proc. VTC2017-Fall, Toronto, Canada September 24-27, 2017,

[13] A. Hussain, N.H. Phulpoto U. Rajput, F. Abbas, and Z. A. Baloch, "Non-Linear energy harvesting Dual-hop DF relaying system over $\eta-\mu$ fading channels", International Journal of Advanced Computer Science and Applications (IJACSA), vol.9, no.6, pp.423-426, 2018.

[14] A. Hussain, S.-H. Kim, and S.-H, Chang, "Dual-hop variable-gain AF relaying with beamforming over $\kappa-\mu$ shadowed fading channels", proc. IEEE GLOBECOM 2016, Washington DC, USA, December 6-8, 2016.

[15] A. Hussain, S.-H. Kim, and S.-H. Chang, "On the performance of dualhop variable-gain $A F$ relaying with beamforming over $\eta-\mu$ fading channels", IEICE Transactions on Communications, vol.E100.B, no.4, pp.619-626, 2017. 